\title{
A case of adrenal Cushing's syndrome with bilateral adrenal masses
}

\author{
Ya-Wun Guo1,2, Chii-Min Hwu2,3, Justin Ging-Shing Won2,3, Chia-Huei Chu3,4 and \\ Liang-Yu Lin ${ }^{2,3}$ \\ 'Department of Medicine, Taipei City Hospital, Zhongxing Branch, Taipei, Taiwan, ${ }^{2}$ Department of \\ Medicine, Division of Endocrinology and Metabolism, Taipei Veterans General Hospital, Taipei, Taiwan, \\ ${ }^{3}$ Faculty of Medicine, National Yang-Ming University School of Medicine, Taipei, Taiwan, and \\ ${ }^{4}$ Department of Otorhinolaryngology-Head and Neck Surgery, Division of Otology, Taipei Veterans \\ General Hospital, Taipei, Taiwan
}

Correspondence

should be addressed

to Liang-Yu Lin

Email

linly@vghtpe.gov.tw

\section{Summary}

A functional lesion in corticotrophin (ACTH)-independent Cushing's syndrome is difficult to distinguish from lesions of bilateral adrenal masses. Methods for distinguishing these lesions include adrenal venous sampling and ${ }^{131 \mid-6 \beta-}$ iodomethyl-19-norcholesterol (131|-NP-59) scintigraphy. We present a case of a 29-year-old Han Chinese female patient with a history of hypercholesterolaemia and polycystic ovary syndrome. She presented with a 6 month history of an $8 \mathrm{~kg}$ body weight gain and gradual rounding of the face. Serial examinations revealed loss of circadian rhythm of cortisol, elevated urinary free-cortisol level and undetectable ACTH level $(<5 \mathrm{pg} / \mathrm{mL})$. No suppression was observed in both the low- and high-dose dexamethasone suppression tests. Adrenal computed tomography revealed bilateral adrenal masses. Adrenal venous sampling was performed, and the right-to-left lateralisation ratio was 14.29. The finding from adrenal scintigraphy with NP-59 was consistent with right adrenal adenoma. The patient underwent laparoscopic right adrenalectomy, and the pathology report showed adrenocortical adenoma. Her postoperative cortisol level was $3.2 \mu \mathrm{g} / \mathrm{dL}$, and her Cushingoid appearance improved. In sum, both adrenal venous sampling and ${ }^{131} \mid \mathrm{IP}-59$ scintigraphy are good diagnostic methods for Cushing's syndrome presenting with bilateral adrenal masses.

\section{Learning points:}

- The clinical presentation of Cushing' syndrome includes symptoms and signs of fat redistribution and protein-wasting features.

- The diagnosis of patients with ACTH-independent Cushing's syndrome with bilateral adrenal masses is challenging for localisation of the lesion.

- Both adrenal venous sampling and ${ }^{131}$ |-NP-59 scintigraphy are good methods to use in these patients with Cushing's syndrome presenting with bilateral adrenal masses.

\section{Background}

Cushing's syndrome results from prolonged, inappropriate exposure to excessive amounts of circulating free cortisol. Endogenous Cushing's syndrome is classified as either corticotrophin (ACTH) dependent or ACTH independent (1). The two most common aetiologies of ACTHindependent Cushing's syndrome are adrenal adenoma and carcinoma. Adrenal adenomas are responsible for approximately $10-15 \%$ of cases of Cushing's syndrome, and adrenal carcinomas are responsible for $<5 \%$ of cases of Cushing's syndrome. The onset of clinical features is gradual in patients with adenomas but often rapid in those with adrenal carcinoma. Other rare causes include 
primary bilateral macronodular adrenal hyperplasia, primary pigmented nodular adrenal hyperplasia and McCune-Albright syndrome (1).

Management of patients with ACTH-independent Cushing's syndrome with bilateral adrenal masses is difficult. Adrenal venous sampling and ${ }^{131}$ I-6 $\beta$-iodomethyl19-norcholesterol (131I-NP-59) scintigraphy have been used to identify functional lesions (2). Herein we present a case of a woman with Cushing's syndrome and bilateral adrenal masses.

\section{Case presentation}

A 29-year-old Han Chinese woman was admitted for evaluation of Cushing's syndrome. She presented with complaints of weight gain of approximately $8 \mathrm{~kg}$ (body height, $163 \mathrm{~cm}$; body weight, $60 \mathrm{~kg} ; \mathrm{BMI}, 22.6 \mathrm{~kg} / \mathrm{m}^{2}$ ) and gradual rounding of her face over the past 6 months. An increasing waist circumference was also observed. She showed no sign of easy bruising, thinning of the skin, supraclavicular fat pads, a buffalo hump or purple striae. She reported a previous history of hypercholesterolaemia and polycystic ovary syndrome. She visited our outpatient department for laboratory examinations, which revealed a morning cortisol level of $17.8 \mu \mathrm{g} / \mathrm{dL}$ (reference range: $5-25 \mu \mathrm{g} / \mathrm{dL}$ ), ACTH level of $<5 \mathrm{pg} / \mathrm{mL}$ (reference range: $<46 \mathrm{pg} / \mathrm{mL}$ ) and urinary freecortisol level of $486 \mu \mathrm{g} /$ day (reference range: $20-80 \mu \mathrm{g} /$ day). She was subsequently admitted.

On physical examination, she had a healthy weight (BMI, $22.3 \mathrm{~kg} / \mathrm{m}^{2}$ ), mildly rounded face, facial plethora

Table 1 Baseline clinical and biochemical characteristics of the patients.

\begin{tabular}{|c|c|c|}
\hline Test & Patient value & Reference range \\
\hline WBC (/cumm) & 6900 & $6900-15800$ \\
\hline $\mathrm{Hb}(\mathrm{g} / \mathrm{dL})$ & 15.5 & $10.2-15.0$ \\
\hline Hct $(\%)$ & 45.3 & $30.1-43.6$ \\
\hline PLT (/cumm) & $191 \mathrm{~K}$ & $152-306 \mathrm{~K}$ \\
\hline BUN (mg/dL) & 10 & $20-68$ \\
\hline Cre (mg/dL) & 0.69 & $1.3-3.3$ \\
\hline ALT (U/L) & 23 & $15-41$ \\
\hline $\mathrm{Na}(\mathrm{mmol} / \mathrm{L})$ & 144 & $126-137$ \\
\hline $\mathrm{K}(\mathrm{mmol} / \mathrm{L})$ & 4.0 & $3.8-5.2$ \\
\hline Cholesterol (mg/dL) & 264 & $125-240$ \\
\hline LDL-c (mg/dL) & 177 & $<160$ \\
\hline $\mathrm{Tg}(\mathrm{mg} / \mathrm{dL})$ & 108 & $20-200$ \\
\hline
\end{tabular}

WBC, white blood cell count; Hb, hemoglobin; Hct, hematocrit; PLT, platelets; BUN, blood urea nitrogen; Cre, creatine; ALT, alanine aminotransferase; $\mathrm{Na}$, sodium; K, potassium; LDL-c: low-density lipoprotein cholesterol; Tg: triglyceride. and ecchymosis of the extremities. Other aspects were unremarkable. The results of a complete blood cell count were within the normal limits, and general biochemical findings showed hypercholesterolaemia (Table 1).

\section{Investigations}

Serial examinations for Cushing's syndrome were then performed. The circadian rhythm of plasma cortisol disappeared $(14.3$ and $14.0 \mu \mathrm{g} / \mathrm{dL}$ at $08: 00 \mathrm{~h}$, and 14.5 and $14.6 \mu \mathrm{g} / \mathrm{dL}$ at $10: 00 \mathrm{~h}$ respectively; reference ranges: cortisol at $08: 00 \mathrm{~h}, 5-25 \mu \mathrm{g} / \mathrm{dL}$; cortisol at 10:00h, $<5 \mu \mathrm{g} / \mathrm{dL})$. Elevated urinary free-cortisol levels were noted (1027 and 958 $\mu \mathrm{g} /$ day respectively), and the ACTH level was undetectable $(<5.0 \mathrm{pg} / \mathrm{mL})$. A 48 -h low-dose dexamethasone test was performed after administration of dexamethasone at $0.5 \mathrm{mg}$ every $6 \mathrm{~h}$ for $48 \mathrm{~h}$. The test results showed a plasma cortisol level of $16.4 \mu \mathrm{g} / \mathrm{dL}$ (cut-off point, $<1.8 \mu \mathrm{g} /$ $\mathrm{dL}$ ) and a urinary free-cortisol level of $1502 \mu \mathrm{g} /$ day (cut-off point, a decrease of $>90 \%$ ). A high-dose dexamethasone suppression test was also performed with administration of dexamethasone at $2 \mathrm{mg}$ every $6 \mathrm{~h}$ for $48 \mathrm{~h}$. The test results showed a urinary free-cortisol level of $1035 \mu \mathrm{g} /$ day (cut-off point, a decrease of $>50 \%$ ). These results were compatible with ACTH-independent Cushing's syndrome.
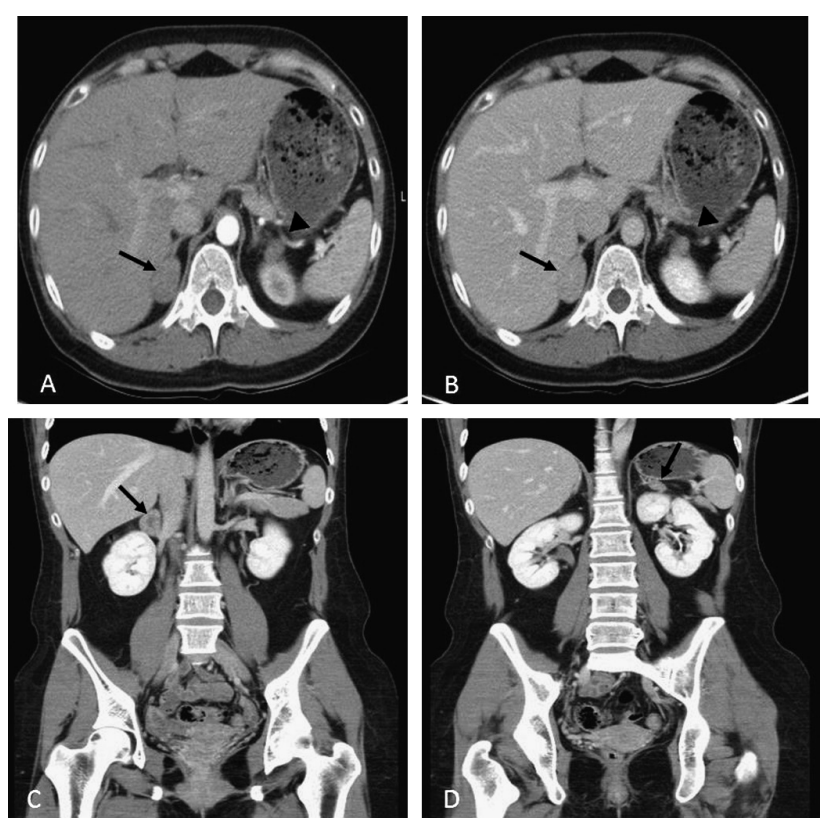

Figure 1

Adrenal CT. (A) Pre-contrast CT showed bilateral adrenal masses (arrow and arrowhead). (B) Post-contrast $\mathrm{CT}$ revealed bilateral adrenal masses (arrow and arrowhead). (C) A right adrenal mass was noted in the coronal view of adrenal CT (arrow). (D) A left adrenal mass was seen in the coronal view of adrenal CT (arrow). 
Table 2 Results of adrenal venous sampling test.

\begin{tabular}{|c|c|c|c|}
\hline & $\begin{array}{c}\text { Right } \\
\text { adrenal } \\
\text { vein }\end{array}$ & $\begin{array}{c}\text { Left adrenal } \\
\text { vein }\end{array}$ & $\begin{array}{c}\text { Inferior } \\
\text { vena cava }\end{array}$ \\
\hline Cortisol ( $\mu \mathrm{g} / \mathrm{dL})$ & 410.26 & 28.7 & 16.8 \\
\hline AV/PV ratio & 24.4 & 1.70 & \\
\hline Cortisol ratio* & 14.29 & & \\
\hline $\begin{array}{l}\text { Catecholamine }(\mathrm{pg} / \mathrm{mL}) \\
\text { (epinephrine/ } \\
\text { norepinephrine) }\end{array}$ & $274 / 330$ & $>2000 / 219$ & $15 / 101$ \\
\hline Aldosterone $(\mathrm{pg} / \mathrm{mL})$ & 694 & 877 & 49.11 \\
\hline
\end{tabular}

* Lateralization ratio: Right adrenal vein cortisol level divided by left adrenal vein cortisol level.AV, adrenal vein; PV, peripheral vein.

Adrenal contrast-enhanced computed tomography (CT) revealed bilateral, round and smooth-contoured nodules, measuring $3 \mathrm{~cm}$ at the medial limb on the right side and $1.5 \mathrm{~cm}$ at the lateral limb on the left side (Fig. 1). In addition, both adrenal nodules showed low unenhanced CT attenuation values $(<10$ Hounsfield units) with rapid contrast medium washout. Either or both of the lesions could have been the cause of cortisol hypersecretion; however, this could not be ascertained using CT. To identify the source of the excess cortisol secretion, we performed adrenal venous sampling after dexamethasone administration. Plasma adrenaline, cortisol and aldosterone levels were measured from the inferior vena cava, left renal vein and bilateral adrenal veins. Successful catheterisation was confirmed on the basis of the following plasma adrenaline levels: right adrenal vein, $274 \mathrm{pg} / \mathrm{mL}$ and left adrenal vein, $>2000 \mathrm{pg} / \mathrm{mL}$ (inferior vena cava adrenaline, $15 \mathrm{pg} / \mathrm{mL}$; Table 2). The adrenal vein-to-peripheral vein cortisol ratio (AV/PV gradient) was 24.4 on the right side and 1.70 on the left side, with a right-to-left lateralisation ratio of 14.29 (Table 2).

The patient also underwent 131 I-NP-59 scintigraphy. Lugol's solution (eight drips per day) and dexamethasone ( $2 \mathrm{mg}$ every $6 \mathrm{~h}$ starting 3 days before the radioactive isotope administration) were administered for a total of 6 days. Tc-99m dimercaptosuccinic acid (DMSA) was then administered for kidney localisation. After $3 \mathrm{~h}$, ${ }^{131}$ I-NP-59 was injected intravenously, and sequential posterior images of the abdomen were obtained. The right adrenal gland was visualised on days 2, 3 and 4. However, the left adrenal gland was not visualised throughout the course of the study. According to the results of the adrenal venous sampling and ${ }^{131} \mathrm{I}-\mathrm{NP}-59$ scintigraphy, we diagnosed the right adrenal adenoma to be a functional mass (Fig. 2).
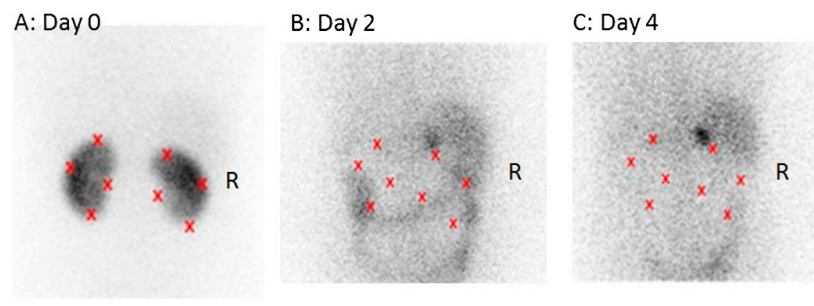

Figure 2

Results of ${ }^{131}$ I-NP-59 scintigraphy. (A) Localisation of bilateral kidneys on day 0 from the posterior view. ( $B$ and $C$ ) Uptake in the right supraadrenal area was clearer during the period (days 2 and 4 ) from the posterior view.

\section{Treatment}

The patient underwent laparoscopic right adrenalectomy in March 2013. The pathology report revealed an adrenal gland of approximately $3 \mathrm{~cm}$. Histological examination revealed an adrenal adenoma measuring approximately $1.2 \times 1 \mathrm{~cm}$, without malignant features. Postoperative steroid supplementation was initiated for her fatigue and low systolic blood pressure of $90-100 \mathrm{mmHg}$ under the impression of postsurgical adrenal insufficiency. On the fourth day after surgical intervention, her postoperative cortisol level at 08:00 h was $3.2 \mu \mathrm{g} / \mathrm{dL}$, without hydrocortisone intravenous supplementation for $>24 \mathrm{~h}$.

\section{Outcome and follow-up}

The patient was regularly followed up at our endocrine department with discontinuation of the steroid supplementation 5 months after surgery. Her body weight was $51 \mathrm{~kg} 8$ months after laparoscopic right adrenalectomy.

\section{Discussion}

If a patient presents with bilateral adrenal masses, it is important to distinguish whether one or both masses are hormonally hyperactive. In patients with bilateral adrenal masses and ACTH-independent Cushing's syndrome, the possible aetiologies include primary bilateral macronodular adrenal hyperplasia, bilateral cortisol secretion adenoma, or single adenoma with a contralateral non-functioning adenoma $(1,2)$. Morphological imaging modalities, including CT and magnetic resonance imaging, are not useful for distinguishing between a hormonally hyperactive and non-functioning adrenal mass (2), whereas both ${ }^{131}$ I-NP-59 scintigraphy and adrenal venous sampling are reportedly useful for identifying adrenal Cushing's syndrome (2). Adrenal 
vein sampling has been used for subtype classification of primary aldosteronism and is recommended by the Endocrine Society Clinical Practice guidelines (3). Adrenal vein sampling has also been performed in patients with pheochromocytoma with no visible source on crosssectional imaging (4). Only a few studies have reported the use of adrenal venous sampling for Cushing's syndrome with bilateral masses, of which the most well known was conducted by the Mayo Clinic (Rochester, MN, USA) $(2,5,6)$. Young and coworkers proposed cut-off values to determine unilateral or bilateral cortisol hypersecretion based on an assessment of 10 patients. They reported that catheterisation can be considered successful if the serum adrenaline level in the adrenal vein exceeds that in the peripheral vein by $>100 \mathrm{pg} / \mathrm{mL}$. The authors also found that a cortisol level gradient $>6.5$ was consistent with a unilateral cortisol-secreting adenoma and that an AV/PV gradient $\leq 3.3$ was compatible with a non-functioning adenoma. The cortisol lateralisation ratio, which indicates the high- to low-side adrenal vein cortisol gradient, was also used. In all of the cases, a lateralisation ratio $\geq 2.3$ was consistent with a predominant cortisol-hypersecreting mass, and a lateralisation ratio $\leq 2.0$ indicated bilateral cortisol hypersecretion (2). In our case, the AV/PV cortisol gradient was 24.4 on the right side, and the right-to-left lateralisation ratio was 14.29 , which were compatible with the surgical findings. This procedure has some limitations, including difficulty approaching a small right adrenal vein and procedure-related complications (4). Because the complication rate is associated with the level of expertise, it has been suggested that the procedure be performed only by an experienced physician.

Radiopharmaceuticals targeted for the adrenal gland enter hormone synthetic pathways, demonstrate affinity for specific tissue receptors, and provide endocrine function in the target tissue. NP-59 was introduced in the late 1970s and acts as a cholesterol analogue (7). After accumulating in the adrenal cortex, radiopharmaceuticals are esterified without being further metabolised (8), and they provide information on both anatomy and in vivo function of the adrenal gland. According to the scintigraphic pattern, physicians can interpret the type of Cushing's syndrome. If the symmetrical imaging is bilateral, the aetiology is ACTH-dependent Cushing's syndrome, including Cushing's disease and ectopic ACTH syndrome. If the result is asymmetrical, bilateral nodular hyperplasia is considered. When only a unilateral image is obtained, the most common aetiology is adrenal adenoma, which is also suspected if bilateral visualisation is absent (8). In our patient, ${ }^{131}$ I-NP-59 scintigraphy revealed unilateral visualisation compatible with adenoma. 131 I-NP-59 scintigraphy is non-invasive but is seldom used because of the complicated protocol and prolonged imaging time. In addition, ${ }^{131}$ I-NP-59 is not widely available in some countries, including the United States (2).

To the best of our knowledge, no studies have reported direct comparisons between 131I-NP-59 scintigraphy and adrenal venous sampling for Cushing's syndrome. The sensitivity of ${ }^{131} \mathrm{I}-\mathrm{NP}-59$ scintigraphy for adenoma in Cushing's syndrome is reportedly $90-100 \%$ $(9,10)$. The specificity of ${ }^{131}$ I-NP-59 scintigraphy for adrenocortical lesions is approximately $86 \%$ (11). Although scintigraphy requires a 5-to7-day interval after iodocholesterol injection, this non-invasive functional test can be added to virtually any diagnostic evaluation without interfering with other studies. The contraindications for this procedure are pregnancy and breastfeeding (12). The side effects, which include nausea, vomiting, chest discomfort, headache, dizziness, tachycardia, flushing and abdominal pain, are related to the radiopharmaceutical. Furthermore, adrenal vein sampling is used mainly in patients with hyperaldosteronism. Even in high-volume centres with experienced radiologists, the success rate is $70-90 \%$ (13). Adrenal vein sampling is rarely used for the study of hypercortisolism, probably because of the rarity of cases in which adrenal cortisol secretion and bilateral nodules present together. However, it can be a useful test in this group of patients with co-presentation of adrenal cortisol and bilateral nodules (14). The contraindications for the procedure include a bleeding tendency, renal insufficiency, accelerated hypertension and allergy to the contrast medium. The side effects include bleeding, adrenal infarction or haemorrhage and a hematoma at the puncture site (4). In our experience, both adrenal venous sampling and 131-NP-59 scintigraphy can provide the correct location of hypersecreting adrenal tumours. We suggest that if a patient presents with Cushing's syndrome with bilateral adrenal tumours, both adrenal vein sampling and ${ }^{131}$ I-NP-59 scintigraphy should be used to identify the location. However, either examination can provide sufficient information if only one is available.

\section{Conclusion}

This report describes a patient with a Cushingoid appearance and bilateral adrenal masses. Both adrenal venous sampling and ${ }^{131}$ I-NP-59 scintigraphy revealed right-side hypersecretion 
of cortisol. Cushing's syndrome in this patient was controlled after laparoscopic right adrenalectomy.

\section{Declaration of interest}

The authors declare that there is no conflict of interest that could be perceived as prejudicing the impartiality of the research reported.

\section{Funding}

This study was partly supported by research grants V103B-019, V104C-085, V105C-131 and V104E11-004-MY2 to L Y L from Taipei Veterans General Hospital, and by research grant MOST 103-2314-B-075-005-MY2 to L Y L from Taiwan's Ministry of Science and Technology, Taipei City, Taiwan. This study was done in Clinical Research Core Laboratory and Medical Sciences and Technology Building in Taipei Veterans General Hospital, Taipei City, Taiwan.

\section{Patient consent}

Written informed consent was obtained from the patient for publication of the submitted article and the accompanying images.

\section{Author contribution statement}

Y W G, C M H, J G W and L Y L drafted the manuscript. C H C and L S H performed the data analysis. All authors read and approved the final manuscript.

\section{References}

1 Newell-Price J, Bertagna X, Grossman AB \& Nieman LK 2006 Cushing's syndrome. Lancet 367 1605-1617. (doi:10.1016/S01406736(06)68699-6)

2 Young WF Jr, du Plessis H, Thompson GB, Grant CS, Farley DR, Richards ML, Erickson D, Vella A, Stanson AW, Carney JA et al. 2008 The clinical conundrum of corticotropin-independent autonomous cortisol secretion in patients with bilateral adrenal masses. World Journal of Surgery 32 856-862. (doi:10.1007/ s00268-007-9332-8
3 Funder JW, Carey RM, Fardella C, Gomez-Sanchez CE, Mantero F, Stowasser M, Young WF Jr \& Montori VM 2008 Case detection, diagnosis, and treatment of patients with primary aldosteronism: an endocrine society clinical practice guideline. Journal of Clinical Endocrinology and Metabolism 93 3266-3281. (doi:10.1210/ jc.2008-0104)

4 Daunt N 2005 Adrenal vein sampling: how to make it quick, easy, and successful. Radiographics 25 S143-S158. (doi:10.1148/rg.25si055514)

5 Martins RG, Agrawal R, Berney DM, Reznek R, Matson M, Grossman AB \& Druce MR 2012 Differential diagnosis of adrenocorticotropic hormone-independent Cushing syndrome: role of adrenal venous sampling. Endocrine Practice 18 e153-e157. (doi:10.4158/EP12136.CR)

$6 \mathrm{Ku}$ EJ, Hong AR, Kim YA, Bae JH, Chang MS \& Kim SW 2013 Adrenocorticotropic hormone-independent Cushing syndrome with bilateral cortisol-secreting adenomas. Endocrinology and Metabolism $\mathbf{2 8}$ 133-137. (doi:10.3803/EnM.2013.28.2.133)

7 Sarkar SD, Beierwaltes H, Ice RD, Basmadjian GP, Hetzel KR, Kennedy WP \& Mason MM 1975 A new and superior adrena scanning agent, NP-59. Journal of Nuclear Medicine 16 1038-1042.

8 Avram AM, Fig LM \& Gross MD 2006 Adrenal gland scintigraphy. Seminars in Nuclear Medicine 36 212-227. (doi:10.1053/j. semnuclmed.2006.03.004)

9 Fig LM, Gross MD, Shapiro B, Ehrmann DA, Freitas JE, Schteingart DE, Glazer GM \& Francis IR 1988 Adrenal localization in the adrenocorticotropic hormone-independent Cushing syndrome. Annals of Internal Medicine 109 547-553. (doi:10.7326/0003-4819109-7-547)

$10 \mathrm{Yu}$ KC, Alexander HR, Ziessman HA, Norton JA, Doppman JL, Buell JF, Nieman LK, Cutler GB Jr, Chrousos GP \& Fraker DL 1995 Role of preoperative iodocholesterol scintiscanning in patients undergoing adrenalectomy for Cushing's syndrome. Surgery 118 981-986. (doi:10.1016/S0039-6060(05)80103-5)

11 Fischer M, Vetter H 1986 Adrenal gland scintigraphy. Radiologe 26 181-185.

12 Hay RV \& Gross MD. Scintigraphic imaging of the adrenals and neuroectodermal tumors. In Nuclear Medicine, edn 2, ch 51, pp 820-844. Ed RE Henkin. Philadelphia, PA, USA: Mosby, 2006.r

13 Stewart PM \& Allolio B 2010 Adrenal vein sampling for primary aldosteronism: time for a reality check. Clinical Endocrinology $\mathbf{7 2}$ 146-148. (doi:10.1111/cen.2010.72.issue-2)

14. Builes-Montaño CE Villa-Franco CA Román-Gonzalez A VelezHoyos A \& Echeverri-Isaza S 2015 Adrenal venous sampling in a patient with adrenal Cushing syndrome. Colombia Médica 46 84-87.

Received in final form 20 March 2016

Accepted 18 April 2016 